\title{
Modeling of sorption kinetics: the pseudo-second order equation and the sorbate intraparticle diffusivity
}

\author{
Wojciech Plazinski $\cdot$ Jakub Dziuba • \\ Wladyslaw Rudzinski
}

Received: 22 October 2010/Accepted: 22 February 2013/Published online: 6 March 2013

(c) The Author(s) 2013. This article is published with open access at Springerlink.com

\begin{abstract}
The applicability of the pseudo-second order equation (PSOE) has been explained on the ground of the model assuming that the overall sorption rate is limited by the rate of sorbate diffusion in the pores of sorbent (intraparticle diffusion model). Mathematical expressions have been proposed in order to describe the dependence of the pseudo-second order constant on such parameters as the initial sorbate concentration, the progress of the sorption process and the solid/solution ratio. Further, it has been shown that equilibrium sorption capacities estimated by using PSOE may be much lower than the actual ones: it depends mainly on how the sorption system is close to equilibrium. The values of parameters applied in calculations were taken from the literature and correspond to the biosorption systems designed to remove the heavy metals from the aqueous solution.
\end{abstract}

Keywords Sorption kinetics - Sorption modeling · Intraparticle diffusivity · Pseudo-second order .

Biosorption

\section{List of symbols}

$\begin{array}{ll}r, x & \text { Coordinates in Eqs. (1) and (2) } \\ t & \text { Time } \\ D_{b} & \text { Intraparticle diffusion coefficient } \\ \beta & \text { Constant related to the physical properties } \\ & \text { of sorbent }\end{array}$

W. Plazinski $(\bowtie) \cdot$ W. Rudzinski Institute of Catalysis and Surface Chemistry, Polish Academy of Sciences, ul. Niezapominajek 8, 30-239, Kracow, Poland e-mail: wojtek_plazinski@o2.pl

J. Dziuba · W. Rudzinski

Department of Theoretical Chemistry, Faculty of Chemistry, UMCS, pl. M. Curie-Sklodowskiej 3, 20-031 Lublin, Poland

$\begin{array}{ll}c_{l}, q_{l} & \begin{array}{l}\text { Local concentrations of sorbate within a } \\ \text { particle, in the solution and in the adsor- } \\ \text { bed phase, respectively }\end{array} \\ q(t), q_{e} & \begin{array}{l}\text { Total adsorbed amounts at time } t \text { and at } \\ \text { equilibrium, respectively }\end{array} \\ \beta & \text { Defined in Eq. (5) } \\ \Delta & \text { Defined in Eqs. (8) and (9) } \\ K_{H} & \text { The Henry constant } \\ R_{0}, l & \text { Constants related to the dimension of } \\ c_{i n}, c_{e} & \text { sorbent particles } \\ & \text { The initial and the equilibrium sorbate } \\ k_{2} & \text { concentrations in the bulk solution, } \\ f & \text { respectively } \\ q_{e}^{(C)} & \text { The pseudo-second order constant } \\ A_{i}-E_{i} \text { and } a_{i}-e_{i} & \text { Best-fit parameters in Eqs. (13) and (14) }\end{array}$

\section{Introduction}

The mathematical form of the pseudo-second order equation (PSOE) was first proposed by Blanchard et al. (1984) to describe the kinetics of heavy metal removal by natural zeolites. At present it is the most commonly used mathematical expression applied to description and correlation of the kinetic data monitored in the solid/solution sorption systems (Ho and McKay 1999; Ho 2006a; Febrianto et al. 2009; Gupta and Suhas 2009). The application of PSOE includes the systems containing various kinds of sorbates (heavy metals in both cationic and anionic forms, organic dyes, phenols, etc.) and sorbents (inorganic minerals, activated carbons, raw biomass, etc.). An extraordinary high efficiency of PSOE in correlating very broad spectrum 
of kinetic data originating from diverse systems suggests that this equation can not represent just one kinetic model. Such suggestions have been launched in the past (Rudzinski and Plazinski 2009; Plazinski et al. 2009). The good applicability of this equation is usually associated with the situation when the rate of direct adsorption/ desorption process (seen as a kind of chemical reaction further referred to as "surface reaction") controls the overall sorption kinetics (Plazinski et al. 2009). This is obviously caused by the fact that each known theoretical ground of PSOE is based on fundamental theories of surface reactions. So far approaches of this kind have been only those offering the clear theoretical interpretations of PSOE (see papers by Plazinski et al. 2009, Liu and Liu 2008 and Azizian 2008). One of the earliest theoretical interpretation of PSOE is that proposed by Azizian (2004). That derivation of PSOE was based on the classical Langmuir model of adsorption kinetics in which adsorption is seen as a chemical reaction occurring on the energetically homogeneous solid surface. A similar approach was presented by Liu and Shen (2008) who showed that the classical Langmuir kinetics can be transformed to a polynomial expression depending on a difference between the equilibrium and the actual (time-dependent) adsorbed amount. Both these papers proved that the Langmuir kinetics can reduce either to the pseudo-first or to the -second order rate equations. In the approaches proposed by Rudzinski and Plazinski (Plazinski et al. 2009), PSOE is treated as a special case of more general kinetic equations, developed for the model of both homogeneous and heterogeneous solid surfaces by using the statistical rate theory approach (Rudzinski and Plazinski 2006, 2009). PSOE cannot be obtained from the SRT approach by the direct, mathematical (symbolic) derivation, nevertheless, the convergence of both these models is, in most cases, nearly perfect as shown by numerical calculations. The other attempts to interpret PSOE went through searching some more general kinetic expressions reducing to both the PSOEs and the pseudo-first order equations under certain conditions (Özer 2007; Brouers and Sotolongo-Costa 2006).

The above-mentioned interpretations can hardly be acknowledged as the complete description of theoretical grounds of PSOE. The reasons for that are following:

(1) Numerous papers reporting the good applicability of PSOE for the data monitored in systems in which kinetic processes other than surface reaction play a significant role (compare Yang and Volesky 1999; Febrianto et al. 2009, for instance).

(2) PSOE may not be interpretable on the basis of many other models in terms of the direct, mathematical (symbolic) derivation. This fact might cause that PSOE has not yet been recognized as the expression predicting the behaviour close to that characteristic of other popular models.

The study described here concerns the interpretation of PSOE in terms of intraparticle diffusion model (IDM). This model assumes that the overall sorption rate is controlled by the rate of sorbate diffusion within the sorbent pores (Suzuki 1990; Filippov and Filippova 1996). It is usually expressed by using partial differential equations but, under certain assumptions, an analytical expression for the timedependent adsorbed amount can be also derived (further description of the accepted details is given in the Sect. 2).

The first aim of this study was to check if PSOE is able to simulate the behaviour predicted by IDM well (both these approaches were represented by the integral forms of respective equations).

The second aim was to find a connection between the parameters appearing in PSOE and those characteristic of IDM. The obtained mathematical dependencies and their features were next analyzed during the simple model investigations and the analysis of the experimental data.

\section{Theory}

The description of sorbate intraparticle diffusion into the porous particle is based on the Fick's laws of diffusion. Its principles are very well known in literature (Crank 1975; Suzuki 1990; McGregor et al. 1965; Mittal et al. 2005; Gupta et al. 2006) but for further consideration, let us recall the most fundamental issues.

The process of sorbate diffusion inside the spherical sorbent particles can be generally described by the following mass-balance equation using the radial coordinate $r$ :

$\frac{\partial c_{l}}{\partial t}=D_{b}\left(\frac{\partial^{2} c_{l}}{\partial r^{2}}+\frac{2}{r} \frac{\partial c_{l}}{\partial r}\right)-\sigma \frac{\partial q_{l}}{\partial t}$,

where $t$ is the time, $D_{b}$ is the diffusion coefficient of sorbate in the particle and $\sigma$ is the constant related to the physical properties of sorbent; it is usually equal to the density/ porosity ratio. The local concentrations of sorbate within a particle, $c_{l}$ (in the solution) and $q_{l}$ (in the adsorbed phase) can be related to their equilibrium relationship or connected by a rate equation when the kinetics of direct adsorption/desorption process is considered (Plazinski and Rudzinski 2010; Bahr and Rubin 1987). Here, only this former case is taken into account as being the most common one. Other kinetic processes (external film diffusion, surface reaction, surface diffusion) are neglected, mainly due to the uncertain values of the crucial parameters (e.g. surface diffusivity). Furthermore, the surface reaction case has been considered elsewhere (Plazinski and Rudzinski 2010). The analogous mass-balance equations taking into 
account such processes can be found elsewhere (see Plazinski and Plazinska 2012 and references therein).

The second frequently used model is based on the concept of sorbent particles having a plane shape. This kind of geometry might be particularly interesting when considering the systems containing seaweed biomass (which are more like flat chips rather than spherical particles) (Yang and Volesky 1999). The appropriate equation has the following form:

$\frac{\partial c_{l}}{\partial t}=D_{b} \frac{\partial^{2} c_{l}}{\partial x^{2}}-\sigma \frac{\partial q_{l}}{\partial t}$,

in which $x$ is the arbitrary position coordinate from the central line of the particle in the thickwise direction. Two dimensions of the particle are assumed to be much greater compared to the third one.

The IDM is defined here as that corresponding to Eqs. (1) and (2) with the following additional limitations:

- all sorbent particles have a uniform size (radius or thickness equal to $R_{0}$ and $2 l$, respectively);

- the initial condition for Eq. (1) corresponding to the experimental conditions (adsorption process) is $q_{l}\left(0 \leq r \leq R_{0}\right)=c_{l}\left(0 \leq r \leq R_{0}\right)=0$ (spherical particles) or $q_{l}(-l \leq x \leq l)=c_{l}(-l \leq x \leq l)=0$ (plane particles) at $t=0$;

- the local equilibrium between the sorbate in the solution and the adsorbed phase can be expressed by the linear Henry's isotherm equation.

Furthermore, it is allowed that the sorbate concentration in the bulk phase is dependent on time.

For the sorption system described by the abovementioned conditions, the two following kinetic equations (2) and (3) were obtained by solving analytically Eq. (1) (Crank 1975). For the spherical particles:

$q(t)=q_{e}\left[1-\sum_{n=1}^{\infty} \frac{6 \beta(\beta+1)}{9+9 \beta+d_{n}^{2} \beta^{2}} \exp \left(-\Delta d_{n}^{2} t\right)\right]$,

and for the plane particles:

$q(t)=q_{e}\left[1-\sum_{n=1}^{\infty} \frac{2 \beta(\beta+1)}{1+\beta+d_{n}^{2} \beta^{2}} \exp \left(-\Delta d_{n}^{2} t\right)\right]$.

$q(t)$ and $q_{e}$ are the total adsorbed amounts at time $t$ and at equilibrium, respectively. The definition of the $\beta$ coefficient is the same for both Eqs. (2) and (4); $\beta$ represents the so-called "adsorbent load factor" defined by using the values of the initial and the equilibrium sorbate concentrations in the bulk solution, i.e. $c_{i n}$ and $c_{e}$, respectively:

$\beta=\frac{c_{e}}{c_{\text {in }}-c_{e}}$.
In other words, this parameter corresponds to the value of the solid/solution ratio of a given system. The higher value of $\beta$ denotes the better efficiency of sorption process (i.e. the lower value of equilibrium sorbate concentration in the bulk solution). The $d_{n}$ 's are $n$th non-zero positive roots of

$\tan d_{n}=\frac{3 d_{n}}{3+\beta d_{n}^{2}}$,

in the case of Eq. (3) (spherical particles), or

$\tan d_{n}=-\beta d_{n}$,

when considering Eq. (4) (plane particles). $\Delta$ also depends on the geometry of particles and (for spherical particles, Eq. (3)) is the following function of $D_{b}, R_{0}, \sigma$ and the Henry constant $\left(K_{H}\right)$ :

$\Delta=\frac{D_{b}}{R_{0}^{2}\left(1+\sigma K_{H}\right)}$,

whereas for the plane particles (Eq. (4)), $\Delta$ takes the form:

$\Delta=\frac{D_{b}}{l^{2}\left(1+\sigma K_{H}\right)}$.

On the contrary to both $q_{e}$ and $\beta$, the value of $\Delta$ depends purely on the physical features of the considered system (such as the sorbate diffusivity or the internal structure of sorbent) but not on the applied operational conditions (i.e. initial solute concentration and mass of sorbent). The mathematical details of obtaining Eqs. (3) and (4) on the basis of more general Eqs. (1) and (2), respectively, can be found elsewhere (Crank 1975).

The most commonly applied form of PSOE is that proposed by Ho and McKay (1998) and based on plotting of $t / q(t)$ against $t$ which should give a linear relationship:

$\frac{t}{q(t)}=\frac{1}{k_{2} q_{e}^{2}}+\frac{t}{q_{e}}$,

$k_{2}$ is the so-called pseudo-second order constant. Alternatively, the nonlinear form of PSOE can be used:

$q(t)=\frac{k_{2} q_{e}^{2} t}{1+k_{2} q_{e}}$.

There exist other linear dependencies corresponding to the PSOE model, as presented by Ho (2006b), which are less frequently used.

The current understanding of PSOE is briefly presented in the Sect. 1.

\section{The scheme of calculations}

Due to the number of parameters $\left(q_{e}, \beta, \Delta\right)$ appearing in Eqs. (3) and (4), a detailed comparative analysis of IDM and PSOE requires considering the following factors. 
(1) One has to know that the ranges of values within $q_{e}, \beta$, $\Delta$ can vary; these ranges should correspond to physical conditions characteristic of a given system or of a certain class of systems. Furthermore, the geometry of particle should be also taken into account.

(2) A significant number of combinations of these parameter values is possible (the total number depends on the accepted number of values of each parameter).

(3) The situation is additionally complicated when taking into account the range of time within which the kinetic measurements are carried out. It is commonly known that the adsorption times monitored in different experiments may differ by several orders of magnitude, depending on the physical nature of an adsorption system. Moreover, the accepted range can have influence on both the obtained values of PSOE parameters and the quality of fits as indicated by the recent theoretical (Rudzinski and Plazinski 2009) and experimental (Derylo-Marczewska et al. 2010) studies. The suggestion for solving this problem is given below.

The above-mentioned factors led to accepting the idea of comparative analysis of PSOE and IDM based on generating multiple sets of the $q(t)$ kinetic data by using Eqs. (3) and (4). Each set corresponds to one unique combination of parameters characterizing a given system. In the next step these data were fitted by PSOE (both its linear and nonlinear forms, i.e. Eqs. (10) and (11), respectively) and the obtained results subjected to further analysis. More details are given below.

For the purpose of expressing the range of time within which the data are generated, we introduce the additional parameter $f$, defined as:

$f=\frac{q\left(t=t_{f}\right)}{q_{e}}$,

where $t_{f}$ is the time at which the equality $q / q_{e}=f$ is fulfilled. The kinetic isotherms of sorption can be treated as monotonic functions, so, the value of $f$ defines $t_{f}$ at the same time. The $f$ parameter can be also described as a degree of reaching the equilibrium state by a sorption system after elapsing of a certain period of time (or as the progress of the sorption process). The value of this parameter can vary within the range $(0 ; 1)$, depending on how close the given system is equilibrium. In our study $t_{f}$ corresponds to the maximum value of time accepted while generating data, which is possible to calculate when $f$ is known. The general idea of using $f$ coefficients is described in more details in Rudzinski and Plazinski (2009).

The values of $q_{e}, \beta, \Delta$ were accepted on the basis of the experimental data reported in the literature treating on the sorption of metal ions by various biosorbents (Febrianto et al. 2009). Typical values of $f$ are between 0.5 and 1 , as it is rather an unusual situation when a significant number of data points is recorded for $f<0.5$. $\beta$ can vary from 0 to $\infty$, however, as shown by Crank (1975), the IDM predictions for the values of $\beta$ above 10 are nearly the same as those for $\beta \rightarrow \infty$. The values of $D_{b}, K_{H}$ and $R_{0}$ (or $l$ ) had to be initially estimated in order to determine the possible values of $\Delta$. It was found that $K_{H}$ can vary in a very broad range (from 1 to $500 \mathrm{~L} / \mathrm{g}$ ), depending on the type of sorption system (Febrianto et al. 2009), whereas in the case of $D_{b}$, it was assumed to be in the range of about $0.1-1$ times the value of the typical diffusivity metal ions in the aqueous solutions (i.e. $\sim 10^{-5} \mathrm{~cm}^{2} / \mathrm{s}$ ) (Rubcumintara and Han 1990). The values of diameter/thickness of the sorbent particles were estimated as $0.2-2 \mathrm{~mm}$. The range of $\Delta$ values was further narrowed, as the preliminary study revealed that small values of $\Delta$ connected with any combination of the remaining parameters lead to the systems with extremely high times required to establish equilibrium (over $100 \mathrm{~h}$ needed to reach $80 \%$ of $q_{e}$ ). When considering real (physical) systems, we assumed that such high values are very unlikely for the accepted kinetic model (IDM) and suggest the co-existence of some other, slower kinetic mechanism such as surface reaction or surface diffusion (Suzuki 1990). The final values of parameters accepted for further investigation are collected in Table 1.

The procedure proposed in order to compare the matching of IDM expressed Eqs. (3) and (4) with PSOE can be divided into the following consecutive steps:

(1) The unique set of four parameter values (taken from Table 1) as well as the sorbent particle geometry were accepted.

(2) The $(t ; q(t))$ data points were generated by using Eqs. (3) or (4). The number of generated points was, in all the cases, equal to 50 and they were located uniformly, i.e. the condition $t_{i+1}-t_{i}=$ constant was always fulfilled for each $i$ th data point. The range of times was dependent on the accepted $f$ value.

(3) Generated sets of data were (a) left unchanged for analysis or (b) subjected to the PSOE linear transformation: $(t ; q) \rightarrow(t ; t / q)$ according to Eq. (10).

Table 1 The values IDM parameters applied during generating the kinetic data by using Eqs. (3) and (4)

\begin{tabular}{lll}
\hline Parameters & Accepted values & Units \\
\hline$q_{e}$ & $5 \times 10^{-4} ; 2 \times 10^{-3} ; 5 \times 10^{-3}$ & $\mathrm{~mol} / \mathrm{g}$ \\
$\beta$ & $0.1 ; 0.5 ; 2 ; 10$ & - \\
$\Delta$ & $1.25 \times 10^{-4} ; 1.25 \times 10^{-5} ; 1.25 \times 10^{-6}$ & $1 / \mathrm{s}$ \\
$f$ & $0.5 ; 0.8 ; 0.99$ & - \\
$\begin{array}{l}\text { Particle } \\
\text { shape }\end{array}$ & Spherical; plane & - \\
\hline
\end{tabular}


(4) The linear (Eq. (10)) and the nonlinear (Eq. (11)) forms of PSOE equation were used to fit the data collected in steps $3 \mathrm{~b}$ and a, respectively; $k_{2}$ was treated as the best-fit parameter. Also, the $q_{e}$ parameter appearing in Eqs. (10) and (11) was treated as an adjustable one and denoted as $q_{e}^{(C)}$; this made it possible to compare its values with the "real" ones, accepted while generating the $(t ; q(t))$ data. Both the obtained best-fit parameters and the corresponding determination coefficients $\left(R^{2}\right)$, were collected.

(5) Steps 1-4 were repeated until the total possible number of combinations of parameter values was reached (i.e. 108 for each particle geometry).

The application of the above-described procedure was enforced by the fact that in the real experiments the possible values of physical parameters can vary in a very wide range. Thus, the present situation corresponds to the analysis of the experimental data collected in 72 different sorption systems with an additional extension, namely, incorporating the $f$ coefficient in order to examine the effect of time range in which the kinetic data are monitored.

The next aim was to find theoretical interpretation of $k_{2}$ and to compare the obtained $q_{e}^{(C)}$ with the accepted $q_{e}$ values. Our attempts proceeded in the following stages:

(1) Accepting a general form of analytical functions $k_{2}\left(q_{e}\right.$, $\beta, \Delta, f)$ and $q_{e}^{(C)}\left(q_{e}, \beta, \Delta, f\right)$. The mathematical form of the function used in both cases can be described by the following two analogical expressions:

$$
\begin{aligned}
& k_{2}=\sum_{i} A_{i} q_{e}^{B_{i}} \beta^{C_{i}} \Delta^{D_{i}} f^{E_{i}}, \\
& q_{e}^{(C)}=\sum_{i} a_{i} q_{e}^{b_{i}} \beta^{c_{i}} \Delta^{d_{i}} f^{e_{i}} .
\end{aligned}
$$

in which $A_{i}-E_{i}$ and $a_{i}-e_{i}$ are the best-fit parameters. The upper limit of the total number of best-fit

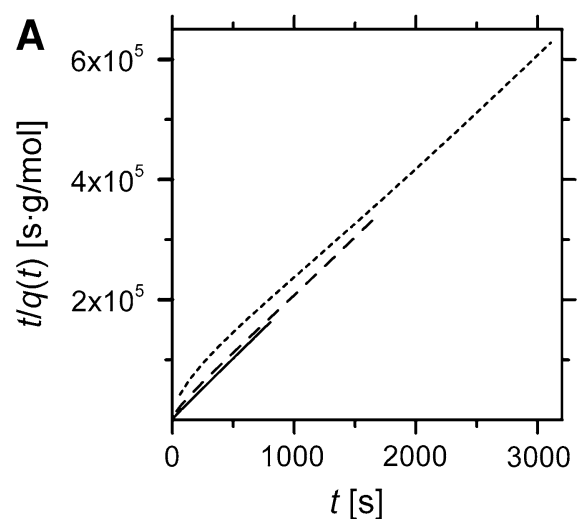

Fig. 1 The example of transformation of the kinetic data generated from Eq. (3) to the linear form of PSOE, i.e. to $t / q(t)$ versus $t$. The calculations were performed for $\Delta=1.25 \times 10^{-4} 1 / \mathrm{s}$ and a $q_{e}=5 \times 10^{-3} \mathrm{~mol} / \mathrm{g}, f=0.99 ; \quad \beta=0.1$ (continuous lines), parameters was set as equal to 9 (this was possible as during optimizing the function the values of some of their best-fit parameters were set to 0,1 or -1 ; this led to decreasing the total number of adjustable parameters).

(2) Searching for the specific mathematical form of functions (13) and (14), able both to describe the desired dependencies well and to maintain the number of best-fit parameters low. The utility of each of the assumed functions was tested in the manner of comparing the "assumed value" with the "predicted value" of the given parameter (i.e. $k_{2}$ or $q_{e}$ ). The arbitrary accepted value of $R^{2}=0.999$ was chosen as the limit above which the function was classified as properly describing the requested dependencies.

(3) Analyzing the obtained dependencies $k_{2}\left(q_{e}, \beta, \Delta\right.$, $f)$ and $q_{e}^{(C)}\left(q_{e}, \beta, \Delta, f\right)$.

All of the fitting calculations presented here were performed using the NonLinearRegress and Regress function of the Statistics 'NonlinearFit' and LinearRegression packages in the Mathematica $5.2^{\mathrm{TM}}$ software.

\section{Results and discussion}

An exemplary plot showing the agreement of IDM and PSOE is presented in Fig. 1. The linear representation of PSOE (Eq. (10)) displays much better agreement with IDM in comparison to Eq. (11). The values of $R^{2}$ vary in the following ranges: $(0.980 ; 1.00)$ (spherical particles, Eq. (10)), (0.969; 1.00) (plane particles, Eq. (6)), (0.957; 0.989) (spherical particles, Eq. (11)) and (0.955; 0.994) (plane particles, Eq. (11)). This observation is in agreement with numerous experimental studies which confirms that

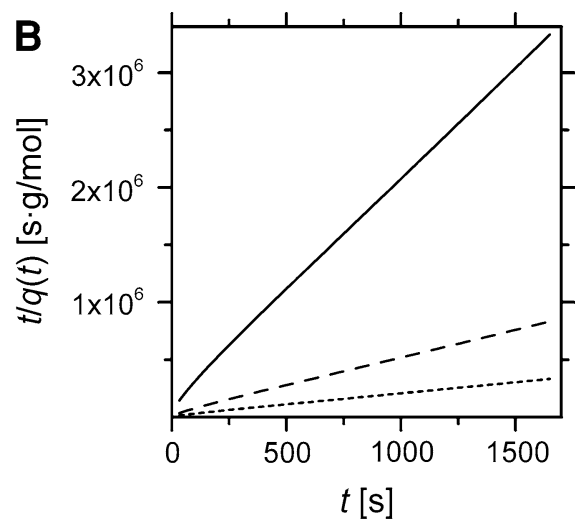

$\beta=2$ (long dashed lines), $\beta=10$ (dashed lines); $\mathbf{b} f=0.99$, $\beta=0.1 ; q_{e}=5 \times 10^{-3} \mathrm{~mol} / \mathrm{g}$ (dashed lines), $q_{e}=2 \times 10^{-3} \mathrm{~mol} / \mathrm{g}$ (long dashed lines), $q_{e}=5 \times 10^{-4} \mathrm{~mol} / \mathrm{g}$ (continuous lines). Linear fits were omitted for the sake of clarity 


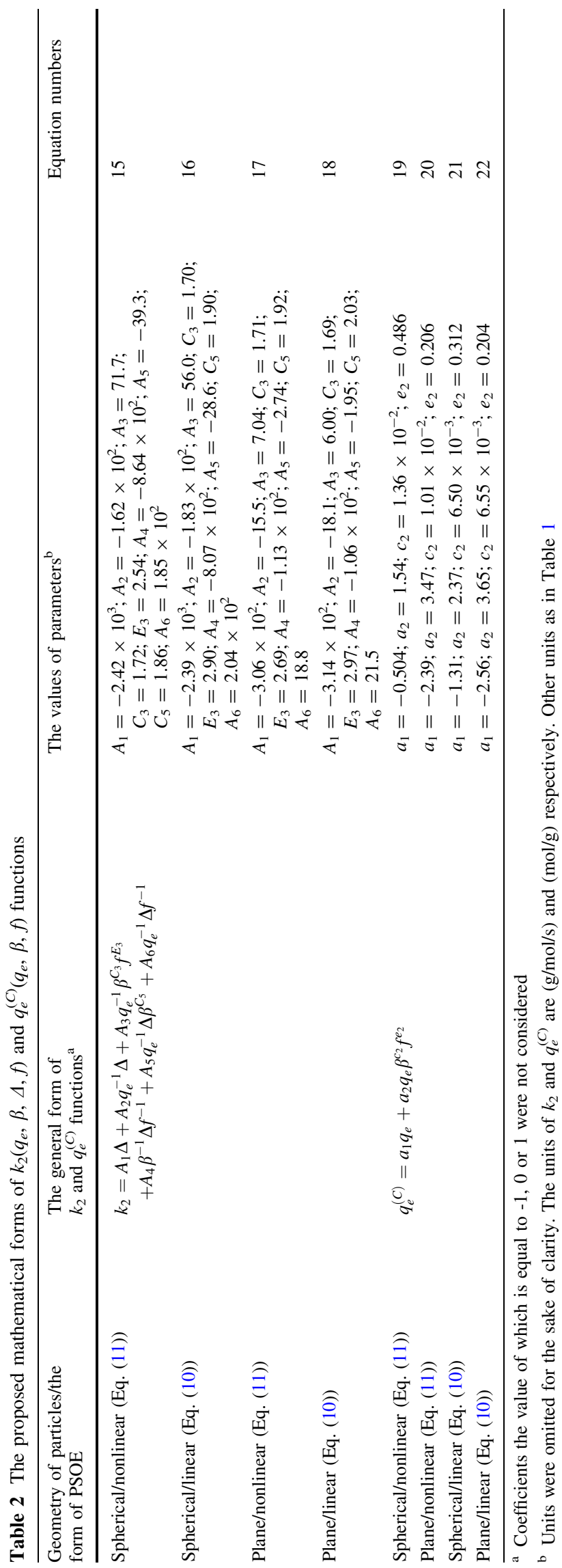

the choice of the form of PSOE can influence both the quality of the obtained fits and the values of adjusted coefficients. For instance, such observation was made by Derylo-Marczewska et al. (2010) who compared the applicability of "classical" (Eq. (10)) and "alternative" PSOE linear plots for description of the sorption kinetics of dyes on mesoporous carbons. The worst correlation was obtained for the systems characterized by low values of $f$, which is true for both considered geometries as well as for both Eqs. (10) and (11). This is also consistent with the results of theoretical (Rudzinski and Plazinski 2009) studies, which show that the applicability of PSOE depends strongly on the ranges of time within which the experimental data are monitored.

The data collected for both linear and nonlinear forms of PSOE were subjected to further analysis as a whole. The agreement between IDM and PSOE (Eq. (11)) appeared not to be as good as that between IDM and PSOE (Eq. (10)), but also these data were kept for comparative purposes.

The specific forms of functions expressed by the general expressions (13)-(14) were proposed, according to the above-described procedure (they are presented in Table 2). It appeared that the same form of the $k_{2}\left(q_{e}, \beta, \Delta, f\right)$ function works well for both forms of PSOE and particle geometry; in the case of $q_{e}^{(C)}\left(q_{e}, \beta, \Delta, f\right)$ the same effect was confirmed. Table 2 contains both the general forms of the obtained functions and the values of best-fit parameters corresponding to the data analyzed in this study (i.e. those generated by using the IDM and parameters collected in Table 1).

The applicability of the found $q_{e}^{(C)}\left(q_{e}, \beta, \Delta, f\right)$ and $k_{2}\left(q_{e}\right.$, $\beta, \Delta, f)$ functions is illustrated in Fig. 2 for the case of spherical particles and the linear form of PSOE. It should be emphasized that the proposed expressions are the empirical functions characteristic of the considered model and the parameters in Table 1. Different departures from these assumptions may result in different values of $a_{i}-e_{i}$ and $A_{i}-E_{i}$ parameters and, eventually, in the mathematical forms of the proposed expressions.

When considering the application of PSOE to correlate the experimental results, one of the most frequently studied questions is how $k_{2}$ depends on the values of the initial sorbate concentration. This was the main reason for performing the study elucidating this problem in terms of IDM and the obtained $k_{2}\left(q_{e}, \beta, \Delta, f\right)$ functions. $c_{i n}$ is not present in $k_{2}\left(q_{e}, \beta, \Delta, f\right)$ directly, but it is one of the most crucial parameters which the value of $q_{e}$ depends on. The following transformation was applied:

$q_{e} \Rightarrow \frac{\beta K_{H} c_{i n}}{1+\beta}$,

in order to obtain the dependence of $k_{2}$ on $c_{i n}$, expressed in terms of IDM. Equation (23) results from the Henry 
Fig. 2 The applicability of the found $q_{e}^{(C)}\left(q_{e}, \beta, \Delta, f\right)(\mathbf{a})$ and $k_{2}\left(q_{e}, \beta, \Delta, f\right)(\mathbf{b})$ functions for the case of spherical particles and the linear form of PSOE. Equations (16) and (21) were applied
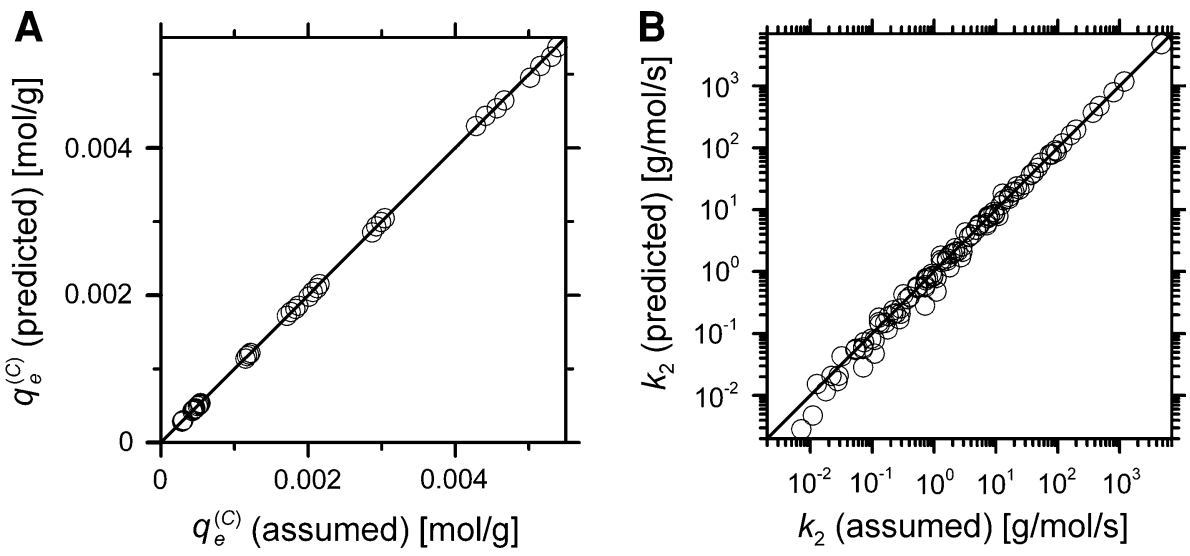

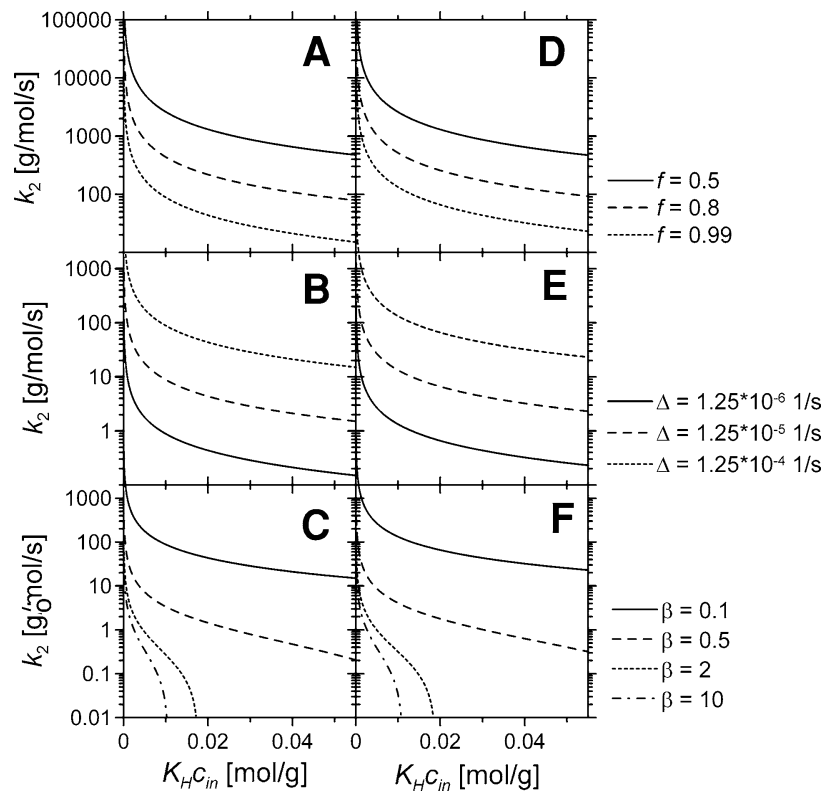

Fig. 3 The $k_{2}\left(q_{e}, \beta, \Delta, f\right)$ functions calculated for the case of spherical particles. If not stated otherwise, the accepted parameters have the following values: $\Delta=1.25 \times 10^{-4} 1 / \mathrm{s} ; f=0.99 ; \beta=0.1$. The non-typical behaviour of $k_{2}$ functions calculated for the two highest values of $\beta$ is obviously the result of inaccuracies in the applied formula, which fails for very low values of $k_{2}$ (this can bee also seen in Fig. 2b). The accepted range of $K_{H} c_{i n}$ corresponds to the values of $q_{e}$ and $\beta$ collected in Table 1 and used in further calculations

adsorption isotherm and from the mass-balance relation (in spite of the fact that $\beta$ is defined through $c_{i n}$ and $c_{e}$, it does not depend on $c_{\text {in }}$ but only on the "mass of sorbent/volume of solution" ratio).

Some of the results of our analysis are presented in Fig. 3 for the case of spherical particles; the results obtained for the plane particles are (qualitatively) the same, so they are not presented. The main findings can be summarized as follows:

(1) The particles geometry seems to be only of secondary importance compared to other factors such as the applied PSOE representation. Nevertheless, one has to appreciate the differences appearing between the coefficients of both $q_{e}^{(C)}\left(q_{e}, \beta, \Delta, f\right)$ and $k_{2}\left(q_{e}, \beta, \Delta\right.$, $f)$ functions.

(2) It appears that the crucial condition for good agreement of PSOE and IDM is applying the linear from of PSOE (Eq. (10)) to transform the kinetic data. However, $k_{2}\left(q_{e}, \beta, \Delta, f\right)$ and $q_{e}^{(C)}\left(q_{e}, \beta, \Delta, f\right)$ functions derived for both linear and nonlinear forms of PSOE (Eqs. (10) and (11), respectively) have the same mathematical form.

(3) The systematic decrease of $k_{2}$ was observed with increasing initial sorbate concentration. This is in agreement with the overwhelming majority of the experimental data (Febrianto et al. 2009). $k_{2}$ usually decreases with increasing $c_{i n}$, which is a fact related to the interpretation of $k_{2}$ as a time-scaling factor (the higher is the $c_{i n}$ value, the longer time is required to reach an equilibrium) (Plazinski et al. 2009). Furthermore, a compact empirical two-parameter formula has been found by Ho and McKay to predict the dependence of $k_{2}$ on $c_{\text {in }}$ satisfactorily well (Ho and McKay 2000). That expression and Eqs. (15)(18) presented here have very similar character (in spite of different algebraic forms) which can be proven by simple comparative analysis (data not shown).

(4) The obtained dependencies of $k_{2}$ on $\Delta$ and $\beta$ are fully consistent with the assumption that $k_{2}$ is a timescaling factor (both increasing of $\beta$ and decreasing of $\Delta$ cause the decrease of sorption rate and, in turn, the values of $k_{2}$ become smaller). This effect can be observed in Fig. 3.

(5) Apart from purely "physical" parameters, $k_{2}$ is also dependent on the range of time in which the kinetic data were monitored (this range is represented here by the value of $f$-see Fig. 3). This finding is similar to that obtained when analyzing PSOE in terms of statistical rate theory (Rudzinski and Plazinski 2009). 
The comparison of the assumed $\left(q_{e}\right)$ and the predicted by using Eqs. (19)-(22) $\left(q_{e}^{(C)}\right)$ equilibrium sorption capacities is presented in Fig. 4 (spherical particles; similar results for the plane particle are not shown). The application of PSOE to determine $q_{e}$ leads to serious deviations, especially when only the initial range of time is considered. The correct values of $q_{e}$ are obtained only for high values of $f$ parameter; this means that, to get the results close to the correct ones, one must have at disposal experimental points measured possibly close to $q_{e}$ (at low values of $f$ one arrives at $q_{e}$ values substantially lower than the actual ones). On the other hand, the accepted value of $\beta$ has only very limited influence on the obtained $q_{e}^{(C)}$. Moreover, the value of $\Delta$ (depending primarily on the sorbate diffusivity) does not practically affect the course of the $q_{e}^{(C)}\left(q_{e}, \beta, \Delta, f\right)$

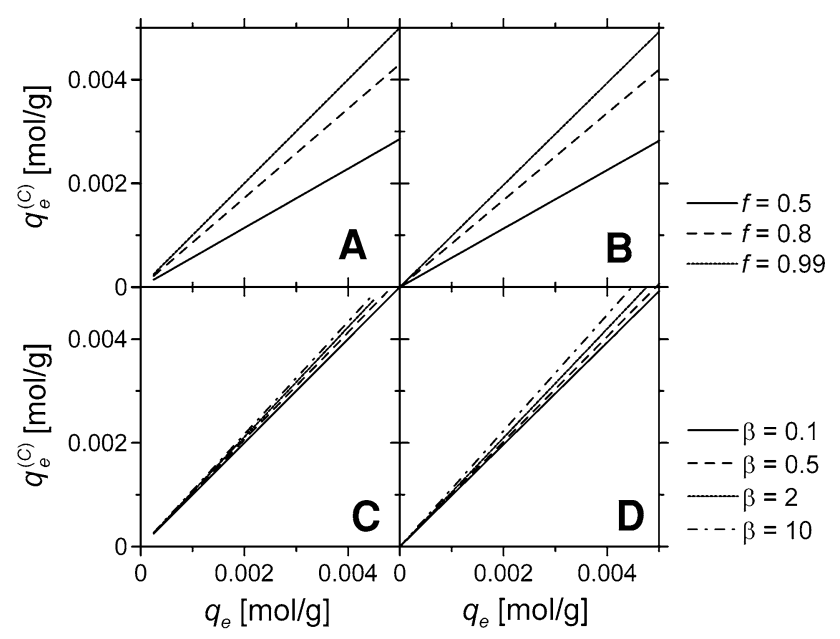

Fig. 4 The $q_{e}^{(C)}\left(q_{e}, \beta, f\right)$ values calculated for the case of spherical particles and plotted in the function of $q_{e}$ (i.e. the "real" equilibrium capacity). If not stated otherwise, the accepted parameters have the following values: $f=0.99 ; \beta=0.1$

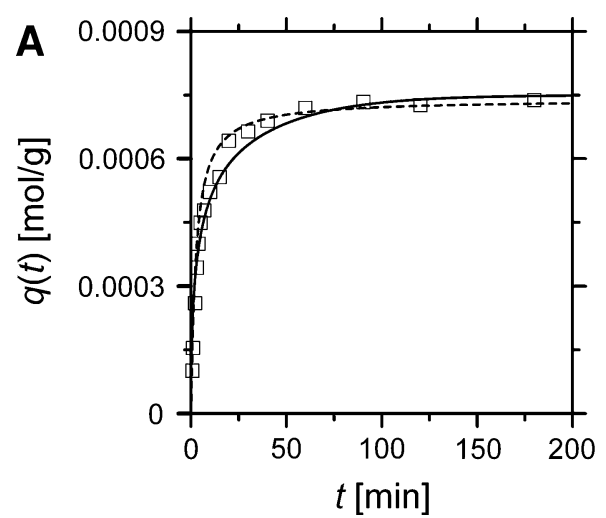

Fig. 5 The agreement of PSOE (broken lines), with the kinetic data reported by Yang and Volesky (squares) and with the IDM (solid lines) represented by Eq. (4). The values of the estimated PSOE constants are equal to: $k_{2}=480.1 \mathrm{~g} / \mathrm{mol} / \mathrm{min}, q_{e}^{(C)}=7.81 \mathrm{mmol} / \mathrm{g}$ function. This is fully understandable, as $\Delta$ in IDM plays a role of time-scaling factor (analogically to the $k_{2}$ constant and PSOE) and its influence on the value of equilibrium parameters is negligible. Moreover, as expected, the $q_{e}^{(C)}$ value depends primarily on the accepted input value of $q_{e}$ (the sum of the $a_{1}$ and $a_{2}$ is always slightly larger than 1).

The applicability of our approach is illustrated by the analysis of the experimental data published by Yang and Volesky (1999). Their paper reports the biosorption of $\mathrm{Cd}^{2+}$ by protonated brown alga Sargassum biomass. All details related to the experimental procedures can be found in the original paper. The system was found to be described well by the IDM and the values of the kinetic (intraparticle diffusivity), equilibrium (monolayer capacity, the Langmuir constant) and technical (solid/solution ratio, the thickness of biosorbent particles) parameters are also known. This let us for estimating the values of parameters appearing in Eqs. (15)-(22) as equal to: $\beta=0.202$, $\Delta=3.73 \times 10^{-3} 1 / \mathrm{min}, \quad q_{e}=0.750 \mathrm{mmol} / \mathrm{g}, f>0.99$ (this latter slight uncertainty does not influence the results). During the next step the values of $k_{2}$ and $q_{e}^{(C)}$ were calculated by applying Eqs. (17), (18) and (21), (22), respectively (the choice of these equations was the result of the sorbent particles geometry). Finally, the obtained PSOE plots were compared with the prediction of IDM, as presented in Fig. 5. The obtained results confirm that (i) PSOE is able to describe the data equally well as the IDM model; (ii) the proposed mathematical expressions (Table 2) predict the values of PSOE-related parameters well.

\section{Concluding remarks}

PSOE can be interpreted in terms of either (a) "surface reaction" models, assuming that the transfer of sorbate

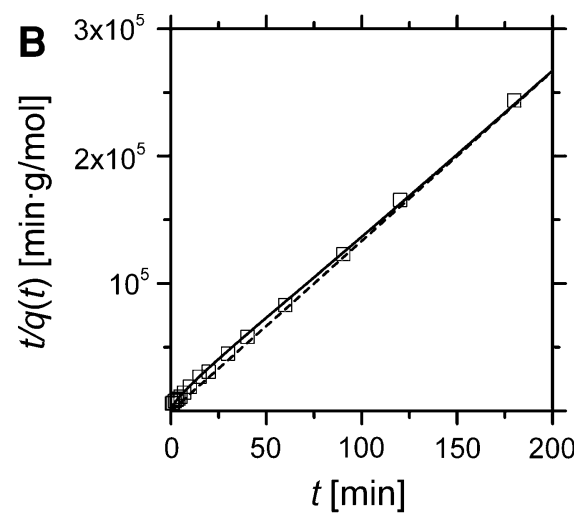

(for the linear form of PSOE) and $k_{2}=529.8 \mathrm{~g} / \mathrm{mol} / \mathrm{min}$, $q_{e}^{(C)}=7.61 \mathrm{mmol} / \mathrm{g}$ (for the nonlinear form of PSOE). Note that broken lines are not fits but PSOE functions (Eqs. (10) and (11)) plotted for the above-mentioned values of $k_{2}$ and $q_{e}^{(C)}$ parameters 
across the solid/solution interface is the slowest step in the whole sorption process or (b) the IDM, as shown in this study. Here, it has been proven that PSOE is a quite flexible mathematical formula, able to simulate the intraparticle diffusion-driven kinetics of sorption for systems with both plane and spherical sorbent particles. The 'mimicking' potential of PSOE is very weakly dependent on both the system-inherent (e.g. diffusivity) and the technical (e.g. the solid/solution ratio) parameters characterizing the sorption system.

Several empirical relationships have been proposed to interpret the PSOE-related coefficients (i.e. $k_{2}$ and $q_{e}^{(C)}$ ) in terms of the physical parameters corresponding to the typical metal ion/algal biosorbent sorption system. The analysis indicated that $k_{2}$ and $q_{e}^{(C)}$ depend primarily, but not exclusively, on the sorbate 'apparent' diffusivities (given by Eqs. (8) and (9)) and on the actual value of $q_{e}$, respectively. The proposed interpretations of the $k_{2}$ and $q_{e}^{(C)}$ constants remain in agreement with the general tendency observed in the experimental data; their testing on the available experimental data (the $\mathrm{Cd}^{2+} /$ Sargassum biomass system) led to satisfactory results.

The degree of agreement of PSOE with IDM and the interpretation of the PSOE-related parameters are strictly connected to the range of times in which the interpreted data were recorded. The applicability of PSOE increases as the system approaches equilibrium which is consistent with the previous studies. However, even when the system is relatively far from equilibrium, PSOE is still able to reproduce the diffusion-driven sorption kinetics very well. As very similar observations were made on the basis of completely different models, one may expect that this kind of behaviour is typical of PSOE. This observation is especially important for the estimation of the equilibrium sorbed amount by using the PSOE, as there may exist significant discrepancies between the actual and estimated $q_{e}$ values, not influencing the quality of the PSOE-related data fit.

Independently of the presented results, our research showed that the interpretation of simple empirical and semi-empirical expressions on the basis of physical models can be done by testing the predicted behaviour for the selected values of parameters characterizing the system. Simple algebraic transformations may not be applicable in the case of more complex models.

Finally, as PSOE is able to follow the behaviour predicted by different models, a simple fitting procedure applied to the kinetic data cannot be therefore treated as a criterion of applicability or inapplicability of the so-called "pseudo-second order model".

Acknowledgments One of the authors (WP) acknowledges the financial support of the Foundation for Polish Science (START Program, 2009 and 2010). The authors (WP and WR) acknowledge the financial support of Polish Ministry of Science and Higher Education (contract financed in 2010-2012 under the Project No. N N204 271238).

Open Access This article is distributed under the terms of the Creative Commons Attribution License which permits any use, distribution, and reproduction in any medium, provided the original author(s) and the source are credited.

\section{References}

Azizian, S.: Kinetic models of sorption: a theoretical analysis. J. Colloid Interface Sci. 276(1), 47-52 (2004)

Azizian, S.: Comments on "Biosorption isotherms, kinetics and thermodynamics" review. Sep. Purif. Technol. 63(2), 249-250 (2008)

Bahr, J.M., Rubin, J.: Direct comparison of kinetic and local equilibrium formulations for solute transport affected by surface reactions. Water Resour. Res. 23(3), 438-452 (1987)

Blanchard, G., Maunaye, M., Martin, G.: Removal of heavy metals from waters by means of natural zeolites. Water Res. 18(12), 1501-1507 (1984)

Brouers, F., Sotolongo-Costa, O.: Generalized fractal kinetics in complex systems (application to biophysics and biotechnology). Physica A 368(1), 165-175 (2006)

Crank, J.: The Mathematics of Diffusion. Clarendon Press, Oxford (1975)

Derylo-Marczewska, A., Marczewski, A.W., Winter, Sz., Sternik, D.: Studies of adsorption equilibria and kinetics in the systems: aqueous solution of dyes-mesoporous carbons. Appl. Surf. Sci. 256(17), 5164-5170 (2010)

Febrianto, J., Kosasih, A.N., Sunarso, J., Ju, Y.-H., Indraswati, N., Ismadji, S.: Equilibrium and kinetic studies in adsorption of heavy metals using biosorbent: a summary of recent studies. J. Hazard. Mater. 162(2-3), 616-645 (2009)

Filippov, L.K., Filippova, N.L.: Overshoots of adsorption kinetics. J. Colloid Interface Sci. 178(2), 571-580 (1996)

Gupta, V.K., Suhas, : Application of low cost adsorbents for dye removal-a review. J. Environ. Manag. 90(8), 2313-2342 (2009)

Gupta, V.K., Mittal, A., Krishanan, L., Mittal, J.: Adsorption treatment and recovery of the hazardous dye, Brilliant Blue FCF, over bottom ash and de-oiled soya. J. Colloid Interface Sci. 293(1), 16-26 (2006)

Ho, Y.S.: Review of second-order models for adsorption systems. J. Hazard. Mater. 136(3), 681-689 (2006a)

Ho, Y.S.: Second-order kinetic model for the sorption of cadmium onto tree fern: a comparison of linear and non-linear methods. Water Res. 40(1), 119-125 (2006b)

Ho, Y.S., McKay, G.: Sorption of dye from aqueous solution by peat. Chem. Eng. J. 70(2), 115-124 (1998)

Ho, Y.S., McKay, G.: Pseudo-second order model for sorption processes. Process Biochem. 34(5), 451-465 (1999)

Ho, Y.S., McKay, G.: The kinetics of sorption of divalent metal ions onto sphagnum moss peat. Water Res. 34(3), 735-742 (2000)

Liu, Y., Liu, Y.-J.: Biosorption isotherms, kinetics and thermodynamics. Sep. Purif. Technol. 61(3), 229-242 (2008)

Liu, Y., Shen, L.: From Langmuir kinetics to first- and second-order rate equations for adsorption. Langmuir 24(20), 11625-11630 (2008)

McGregor, R., Whitney, C.K., Hamilton, R.L.: Applicability of Fick's Law to diffusion and sorption data. Text. Res. J. 35(3), 279 (1965) 
Mittal, A., Kurup, L., Gupta, V.K.: Use of waste materials-bottom ash and de-oiled soya, as potential adsorbents for the removal of Amaranth from aqueous solutions. J. Hazard. Mater. 117(2-3), 171-178 (2005)

Özer, A.: Removal of $\mathrm{Pb}$ (II) ions from aqueous solutions by sulphuric acid-treated wheat bran. J. Hazard. Mater. 141(3), 753-761 (2007)

Plazinski, W., Plazinska, A.: Equilibrium and kinetic modeling of adsorption at solid/solution interfaces. In: Bhatnagar, A. (ed.) Application of Adsorbents for Water Pollution Control. Bentham Science, Sharjah (2012)

Plazinski, W., Rudzinski, W.: A novel two-resistance model for description of the adsorption kinetics onto porous particles. Langmuir 26(2), 802-808 (2010)

Plazinski, W., Rudzinski, W., Plazinska, A.: Theoretical models of sorption kinetics including a surface reaction mechanism: a review. Adv. Colloid Interface Sci. 152(1-2), 2-13 (2009)
Rubcumintara, T., Han, K.N.: The effect of concentration and temperature on diffusivity of metal compounds. Metall. Trans. B 21(3), 429-438 (1990)

Rudzinski, W., Plazinski, W.: Kinetics of solute adsorption at solid/ solution interfaces: a theoretical development of the empirical pseudo-first and pseudo-second order kinetic rate equations, based on applying the statistical rate theory of interfacial transport. J. Phys. Chem. B 110(33), 16514-16525 (2006)

Rudzinski, W., Plazinski, W.: On the applicability of the pseudosecond order equation to represent the kinetics of adsorption at solid/solution interfaces: a theoretical analysis based on the statistical rate theory. Adsorption 15(2), 181-192 (2009)

Suzuki, M.: Adsorption Engineering. Kodansha, Tokyo (1990)

Yang, J., Volesky, B.: Cadmium biosorption rate in protonated Sargassum biomass. Environ. Sci. Technol. 33(5), 751-757 (1999) 